\title{
A NOVEL MODEL OF RF MEMS SHUNT SWITCH
}

\author{
Beneya. $L^{1}$, Praveen Kumar. $S^{2}$ \\ ${ }^{1}$ PG Scholar, Electronics and Communication Engineering, Saveetha Engineering College, Tamil Nadu, India \\ ${ }^{2}$ Associate Professor, Electronics and Communication Engineering, Saveetha Engineering College, Tamil Nadu, India
}

\begin{abstract}
The advancement in the Radio Frequency Micro- Electro Mechanical Systems (RF MEMS) switch has enabled new technologies for microwave switching applications. It is the method used for reduction of material used which in turn decreases the cost and the power dissipation. The RF MEMS shunt switch is constructed for high frequency microwave switching. The switch is driven by electrostatic actuation. The contact line and the RF signal line behaves as the parallel plate capacitor. The Switch provides a good isolation in the off state and less insertion loss. The geometry parameters of the switch is chosen such that the switch can be easily integrated. The Switch Niobium metal is used for the contact lines as it has good superconducting characteristics in cryogenic temperature. The performance of the switch is analyzed and the result shows the deformation of the switch with low actuation voltage.
\end{abstract}

Keywords: RF MEMS, Shunt switch, Superconducting, and Cryogenic.

\section{INTRODUCTION}

The RF MEMS switch is a miniature model which shows good broadband performance. It is constructed for broadcasting of RF signal [1] which will connect the antenna to the transmitter or receiver. By using electrostatic actuation the RF MEMS switch is actuated where as the traditional switches depend on the impedance of the semiconductor device that is actuated by the electric field. The shunt switch has fixed beams where the movable area toggles the switch between the input and the output of the RF signal line.

The Shunt switch is also known as the capacitive switch which is constructed using fixed beams. The principle mechanism of the switch is to make the contact line to toggle with the RF signal line. When the electrostatic force is applied the contact line undergoes a mechanical deformation that will get contact with the RF signal line. When the force is cutoff, due to the elastic property of the material it gains its original shape. In the off state the switch is open circuit and it provides a good isolation for the switch. The isolation of the switch reduces the leakage of RF power in the device. In the on state the switch is short circuit in which the voltage flows through the device that make the contact line to deform to get contact with the signal line.

The geometrical parameter of the switch such as the air gap between the contact line and the signal line provides a good isolation for the device. The RF Power leakage is reduced due to the isolation of the device in the OFF state. The switch does not consume current as the DC voltage required for the switch is very low, hence there is less Power dissipation. The basic switching parameter includes the isolation of the device and the insertion loss with fast switching technique in terms of nano seconds. Since there is no pn junction used the device has less distortion with high linearity. The niobium metal is used for the contact lines as it has superconducting characteristics in cryogenic temperature. These advantages makes the device more suitable for switching application.

\section{DESIGN DESCRIPTION}

\subsection{Equivalent Model of Shunt Switch}

The shunt switch is also known as capacitive switch. The contact line and the RF signal line behaves as the parallel plate capacitor. Hence the electrostatic force generated in the parallel plate is given by

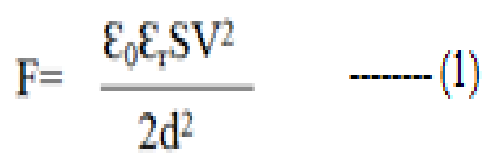

Where, $\quad S-$ Area of Electrode $=30000 \mu \mathrm{m}^{2}$,

$\varepsilon_{0}$ - Dielectric coefficient of vacuum

$=8.854 \times 10^{-12} \mathrm{~F} / \mathrm{m}$

$\varepsilon_{\mathrm{r}}$ - Dielectric coefficient of surrounding air.

The electrostatic force thus obtained is $118 \mu \mathrm{N}$. Beyond this value the metal will lose its elastic property. The force thus obtained makes the metal contact line to snap down unto the RF signal line. This makes the switch to toggle between the ON state and the OFF state. The electrostatic force makes the contact line to snap down on the RF signal line. The elastic property of the metal makes the contact line to gain its original position once when the supply is cutoff. The equivalent circuit of the shunt switch is shown in Fig 1. 


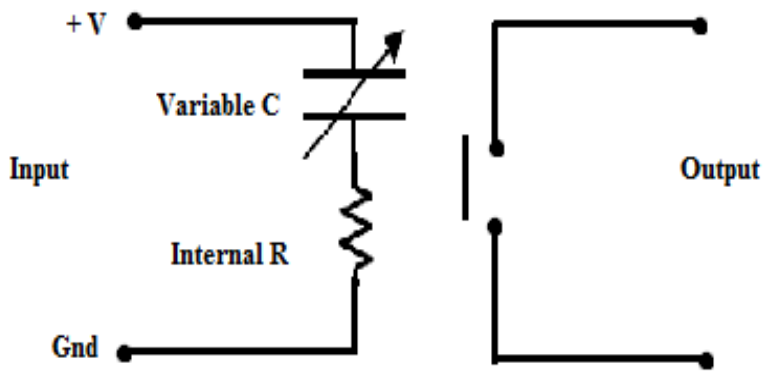

Fig -1: Equivalent Circuit diagram of shunt switch.

The capacitance of the capacitor in the shunt switch is shown in equation (2).

$$
\mathrm{C}=\frac{\varepsilon_{0} \varepsilon_{\mathrm{r}} \mathrm{A}}{\mathrm{t}}
$$

Where C-Capacitance of the shunt switch $(\mathrm{pF})$,

A- Area of the signal line $=10000 \mu \mathrm{m}^{2}$,

$\mathrm{t}$ - thickness of the signal line $=2 \mu \mathrm{m}$,

Thus a capacitance of $44 \mathrm{pF}$ is obtained. The LC resonant frequency of the shunt switch is given in the equation (3)

$$
f_{0}=\frac{1}{2 \pi \sqrt{L C}}
$$

Whereas the range of inductance is in the order of $\mathrm{nH}$ and the capacitance of the shunt switch that operates in the microwave frequency is in the range of $44 \mathrm{pF}$. The resonating frequency is $3.6 \mathrm{GHz}$.

\subsection{Mechanical Model of Shunt Switch}

The shunt switch has fixed - fixed beam in which the contact mechanism of the switch rely on the mechanical deformation of the contact line. Due to the elastic property of the switch it gains its original shape. Typically the contact mechanism requires some voltage for the electrostatic actuation. The theoretical representation of the amount of voltage required is expressed in equation (4).

$$
\mathrm{V}=\sqrt{\frac{8 k}{27 \varepsilon 0 W w} \mathrm{~g} 0^{3}}
$$

Where $\mathrm{k}$ is the spring constant, $\varepsilon_{0}$ is the Permittivity of free space, $\mathrm{W}$ is the width of the RF signal line $(\mu \mathrm{m})$ and $\mathrm{w}$ is the width of the contact line $(\mu \mathrm{m}) . \mathrm{g}_{0}$ is the air gap between the contact line and the signal line. Theoretically the value of actuation voltage thus obtained is $2.012 \mathrm{~V}$. The spring constant is given in equation (5).

$$
\mathrm{k}=32 \operatorname{Ew}\left(\frac{t}{l}\right)^{3}
$$

Where $\mathrm{t}$ - thickness of the beam $=2 \mu \mathrm{m}$;

1- length of the beam $=200 \mu \mathrm{m}$;

E- Young's modulus of $\mathrm{Nb}=105 \mathrm{GPa}$;

From equation (5) it is to be noted that the spring constant depends on the $t$ thickness of the beam. Hence the spring constant $\mathrm{k}$ is a function of the thickness $\mathrm{t}$. The increase in the beam thickness will increase the voltage drastically. The typical value of spring constant is $0.504 \mathrm{~N} / \mathrm{m}$.

The switching speed of the capacitive switch can be obtained from equation (6)

$$
\mathrm{Ts}=3.674 \frac{\mathrm{V}}{\mathrm{V}_{\mathrm{s}} \mathrm{W}_{0}} \quad \ldots
$$

Where $\omega_{0}$ is the angular frequency; $\omega_{0}=2 \pi \mathrm{f}_{0}$ which is in the range of $376.99 \mathrm{~Hz}$. The switching speed thus obtained is 9.8 ns. Hence a fast switching performance is obtained. The skin depth can be calculated from equation (7)

$$
\delta=\frac{1}{(\pi f \mu \sigma)^{0.5}}
$$

Where $\mu$ - permeability of $\mathrm{Nb}=4 \pi \times 10^{-7} \mathrm{H} / \mathrm{m}$, $\sigma$ - electrical conductivity of $\mathrm{Nb}=7.1 \times 10^{-6} \mathrm{~S} / \mathrm{m}$

Hence the skin depth is $0.3 \mu \mathrm{m}$. The $\mathrm{S}$ parameter for which the return loss can be calculated using equation (8)

$$
\mathrm{S}_{11}^{2}=\frac{\omega^{2} \mathrm{C}^{2} \mathrm{Z}_{0}^{2}}{4}
$$

Where $\mathrm{Z}_{0}$ is the impedance $=50 \Omega \mathrm{m}$ and the value of $\mathrm{S}_{11}=$ 13.6dB. The increase in the width of the bridge thus increases the magnitude of $S_{11}$ and decreases $S_{11}$ in decibel. Hence the width should be greater than $100 \mu \mathrm{m}$.

\section{GEOMETRICAL DESCRIPTION}




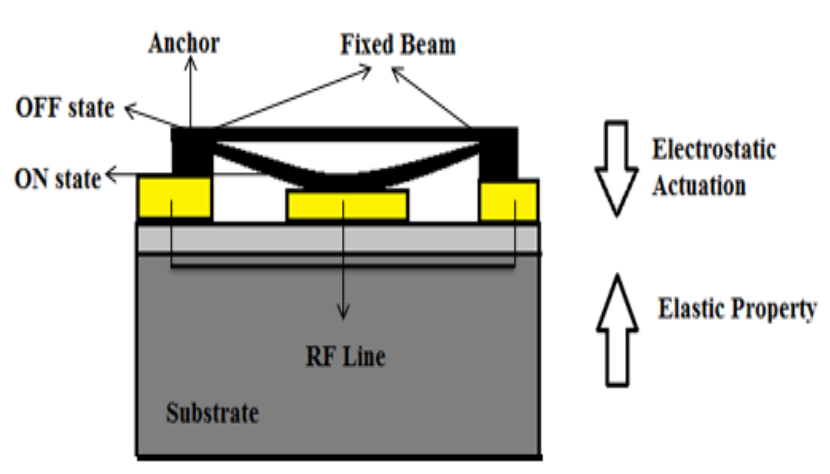

Fig -2: Schematic Structure of Capacitive Switch

The geometrical parameter of the switch is constructed using comsol 4.2a software. In the mechanical structure the solid mechanics is selected. The mechanical structure is studied and the stationary analysis is done to determine the deformation of the device for the required voltage. The dimension of the signal line according to $\mathrm{G} / \mathrm{W} / \mathrm{G}$ is of $60 / 100 / 60 \mu \mathrm{m}$ respectively. The width, height and the thickness of the beam is 150,200 and $2 \mu \mathrm{m}$ respectively. A superconducting metal, niobium is used for the contact line.

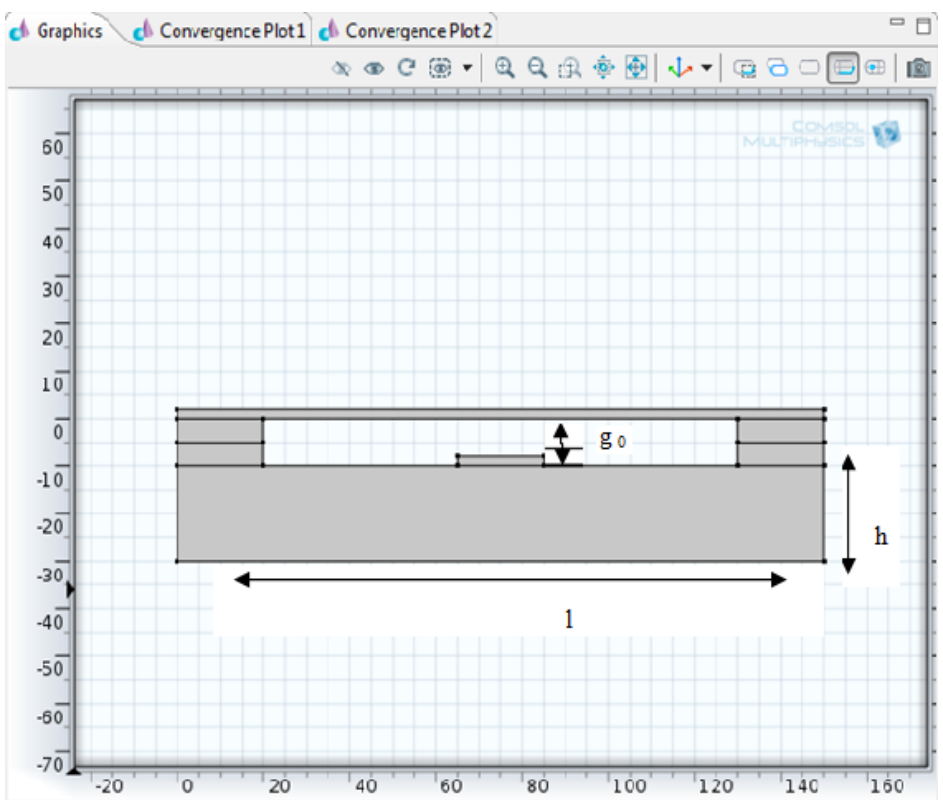

Fig -3: Design description of Capacitive Switch

Once the structure is designed, the material are chosen for all the domain. For the contact line of the capacitive switch niobium metal is chosen. Then the structure is meshed. The meshed structure is shown in Fig - 4.

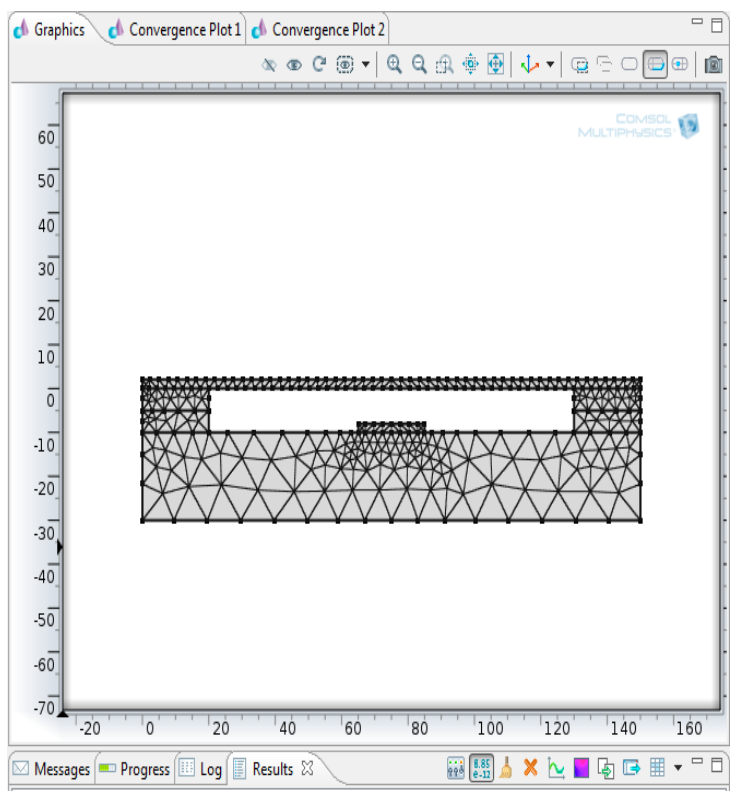

Fig -4: Meshed Structure of Capacitive Switch

\section{STATIC AND MODAL ANALYSIS}

The meshed structure is analyzed by applying the force on the boundary of the contact line. From equation (1) the force exerted on the contact line is $118 \mu \mathrm{N}$. The static study determines the voltage required for the deformation and the spring constants. From equation (2) $3 \mathrm{~V}$ is obtained. The modal analysis determines the frequency of the switch.

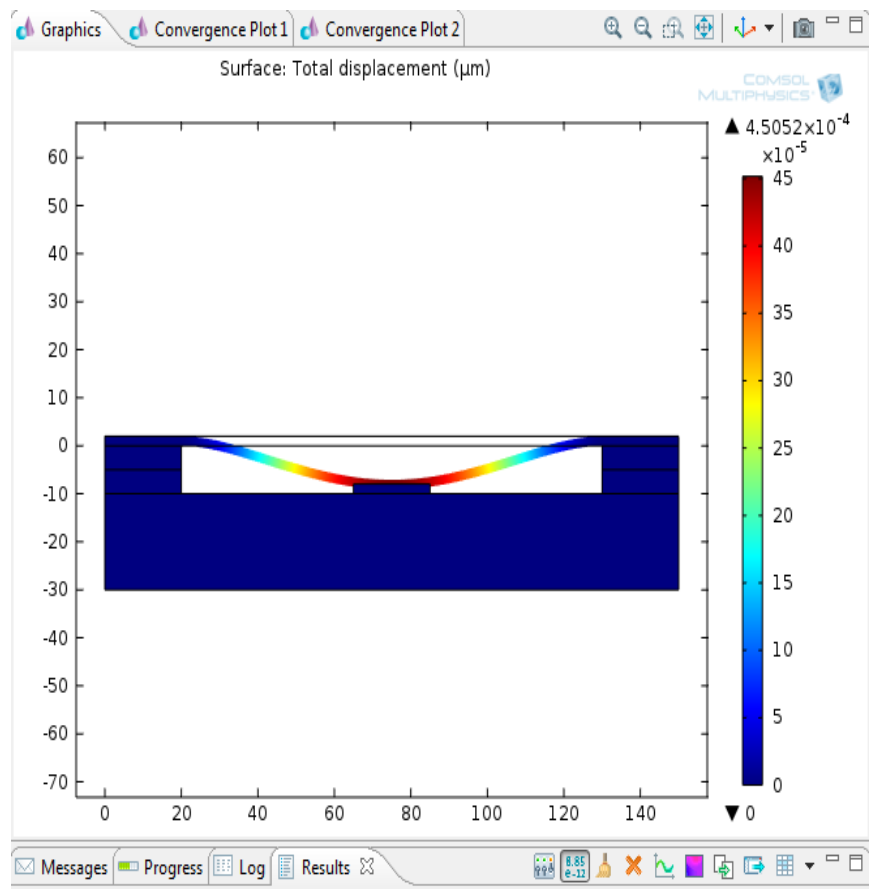

Fig -5: Displacement of the contact line of Capacitive Switch 


\section{RESULTS}

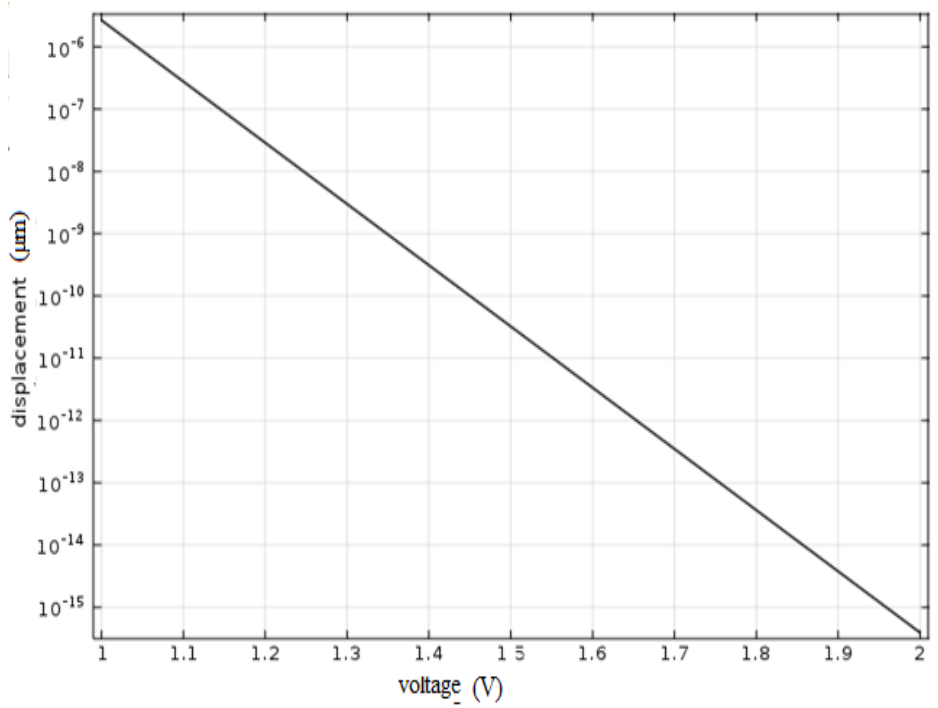

Fig -6: Displacement Vs Voltage Curve

The result shows the deformation of the switch for the applied voltage. A graphical representation of the displacement Vs voltage curve is shown in Fig-6. Hence a linear displacement is obtained for the applied voltage. The simulated result thus obtained determines the actuation voltage of $2 \mathrm{~V}$ which is proved in the equation (4).

\section{CONCLUSIONS}

The designed capacitive switch has high linearity hence can be easily integrated with superconducting micro electronics technology. The shunt switch shows high performance in cryogenic temperature and it has a low actuation voltage in the order of $2.012 \mathrm{~V}$. The frequency is $3.6 \mathrm{GHz}$ which is used to switch Bluetooth devices. The shunt switch exhibits excellent broadband performance and fast switching action by reducing the power consumption.

\section{REFERENCES}

[1] Aditi Sharma; Vivek K. Dwivedi; and G. Singh; "THz Rectangular Microstrip Patch Antenna on Multilayered Substrate for Advance Wireless Communication Systems", Progress In Electromagnetics Research Symposium, Beijing, China, March 23-27, 2009

[2] Baskar.S; Chanemouga priya; Saraswathi.D; hailesh.V; "A Novel Cram of Reconfigurable Patch Antenna using RF MEMS”, IEEE International Conference Digital Object Identifier, 2013.

[3] Brusa E; De Pasuquale G; Soma A; “Experimental Characterization of Electro-Thermo-Mechanical Coupling in Gold RF Microswitches", IEEE journals \& magazines, IEEE transactions on Volume: 22, Issue:4, 2013.
[4] Elsherbeni A.Z; Fan Yang; kai- Fong Lee; Khidre A; "Wide Band Dual-Beam U-Slot Microstrip Antenna", IEEE journals \& magazines, IEEE transactions on Digital Object Identifier, volume 61, issue: 3, 2013.

[5] Raafat R. Mansour; Sara S. Attar, "Low Temperature Superconducting RF MEMS Devices", IEEE journals \& magazines, IEEE transactions on Volume: 23, Issue:3, 2013.

[6] Bedri A. Cetiner; Jofre L; Mopidevi H; Rodrigo D; Unlu M; Zohur A; "RF MEMS Reconfigurable TwoBand Antenna", IEEE antennas and wireless propagation letters, Volume 12, 2013

[7] Verma K.K; Vishwakama R.K; "Electromagnetically coupled square microstrip antenna for dual-band operation ", IEEE International Conference Digital Object Identifier, 2013.

[8] Jie-Ying Tang, Lei Han, Li-Feng Wang, Qing-An Huang, and Yan-Qing Zhu Senior Member, IEEE, "A Novel Three-State RF MEMS Switch for Ultrabroadband (DC-40GHz) Applications" 2013 IEEE electron device letters.

\section{BIOGRAPHIES}

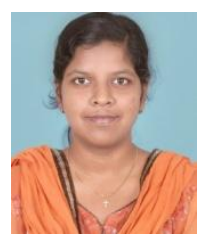

L. Beneya has received the B.E. degree in Electricals and Electronics Engineering in the year 2012 and pursuing M.E. degree in VLSI Design in Saveetha Engineering College. Her area of interest include MEMS and VLSI Design.

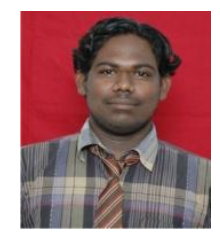

S. Praveen Kumar, M.E.,(Ph.D) Specialized in Applied Electronic. Have 6 years of teaching experience. His Area of interest includes MEMS, BIO-MEMS, WIRELESS SENSORS, Digital Image Processing, VLSI and Wireless Communication. 